\title{
¿Quiénes discriminan a las y los inmigrantes en el Estado de Hidalgo, México? Aproximaciones al perfil desde la Encuesta Nacional sobre Discriminación 2010 y 2017
}

\author{
Who discriminate against immigrants in the State of Hidalgo, Mexico? \\ Approaches to the profile from the National Survey on Discrimination \\ 2010 and 2017
}

Carlos Mejía-Reyes*, Adrián Galindo-Castro

Universidad Autónoma del Estado de Hidalgo, México

* Autor a quien se dirige la correspondencia: mejiareyescarlos@gmail.com

Recibido: 26 de julio de 2018 / Aceptado: 22 de enero de 2019

\section{Resumen}

T a discriminación en México se ha catalogado como un fenómeno social preocupante. Los sectores con mayor Uexclusión, según las encuestas son indígenas, mujeres, homosexuales, lesbianas, personas con discapacidad, jóvenes, adultas (os) mayores, inmigrantes, personas con ideas políticas "distintas", doctrinas religiosas diversas, de clases sociales bajas e inmigrantes. Sin embargo, en algunas regiones del país, estos sectores poblacionales padecen en mayor medida prejuicios que en algunas otras. Así, el Estado de Hidalgo en México, se caracteriza por tener altos niveles de discriminación hacia personas externas a su territorio y en este documento extraemos un perfil analítico del sujeto discriminante a partir de técnicas estadísticas.

Palabras clave: Discriminación, Estado de Hidalgo, México, inmigración, Encuesta Nacional sobre Discriminación

\section{Abstract}

$\mathrm{D}$ iscrimination in Mexico has been classified as a worrying social phenomenon. The most excluded sectors, according the surveys are indigenous, women, homosexuals, lesbians, people with disabilities, young people, adults, immigrants, people with "different" political ideas, diverse religious doctrines, low social classes and immigrants. However, in some regions of the country, these population sectors suffer more prejudice than in some others. Thus, in the State of Hidalgo Mexico, is characterized by having high levels of distinction towards people outside its territory and in this document we extract an analytical profile of the discriminating subject from statistical techniques. 


\section{Introducción}

Dicta la Constitución Política Mexicana (2016) en el artículo primero:

Queda prohibida toda discriminación motivada por origen étnico o nacional, el género, la edad, las discapacidades, la condición social, las condiciones de salud, la religión, las opiniones, las preferencias sexuales, el estado civil o cualquier otra que atente contra la dignidad humana y tenga por objeto anular o menoscabar los derechos y libertades de las personas.

De este párrafo se derivó la creación, en 2003, de la Ley Federal para Prevenir y Eliminar la Discriminación (LFPED) que a su vez en su artículo 16 establece la generación del Consejo Nacional para Prevenir y Eliminar la Discriminación (Conapred). Esta Ley fue reformada el 20 de marzo de 2014, y en ella se define en su Artículo 1, Numeral III la discriminación como:

Para los efectos de esta ley se entenderá por discriminación toda distinción, exclusión, restricción o preferencia que, por acción u omisión, con intención o sin ella, no sea objetiva, racional ni proporcional y tenga por objeto o resultado obstaculizar, restringir, impedir, menoscabar o anular el reconocimiento, goce o ejercicio de los derechos humanos y libertades, cuando se base en uno o más de los siguientes motivos: el origen étnico o nacional, el color de piel, la cultura, el sexo, el género, la edad, las discapacidades, la condición social, económica, de salud o jurídica, la religión, la apariencia física, las características genéticas, la situación migratoria, el embarazo, la lengua, las opiniones, las preferencias sexuales, la identidad o filiación política, el estado civil, la situación familiar, las responsabilidades familiares, el idioma, los antecedentes penales o cualquier otro motivo; También se entenderá como discriminación la homofobia, misoginia, cualquier manifestación de xenofobia, segregación racial, antisemitismo, así como la discriminación racial y otras formas conexas de intolerancia. (Ley Federal para Prevenir y Eliminar la Discriminación, 2014)

Así, la definición de discriminación implica una conducta "culturalmente fundada, sistemática y socialmente extendida" de desprecio, basado en prejuicios negativos contra personas cuya consecuencia es la enajenación sus derechos fundamentales (Rodríguez, 2005).

Si bien, históricamente, en el caso mexicano, la discriminación ha tenido como sujetos principales de menosprecio a los y las indígenas (Bonfil, 1987) se ha demostrado desde los primeros ejercicios cuantitativos, como en la primera Encuesta Nacional sobre Discriminación (Enadis) en 2005, que son diversos los sectores que padecen el prejuicio negativo en el contexto nacional. Sin embargo, en el caso que nos aqueja -el Estado de Hidalgo, México- se presenta un fenómeno particular de interacciones diferenciadas:

Es por ello que pensar en extraños, recién llegados o aquellos que vienen de fuera, no significa atender a acontecimientos novedosos en las relaciones sociales; pero el hecho de que estas categorías formen parte de nuestras preocupaciones y debates coetáneos remite a un horizonte temporal que justifica tal interés cognoscitivo (Sabido, 2012, p. 9).

El extranjero, representa un tipo de acciones recíprocamente orientadas en la compleja red de efectos mutuos que conforman la sociedad (Simmel, 2014) cuya característica se define por la coexistencia de la situación de inclusión y exclusión. "En dicho sentido el extranjero no es una persona sino una forma social, esto es, se trata de un vínculo específico de relación, una forma particular de ser con otros" (Sabido, 2012, p. 11). Es decir que ser extranjero no necesariamente significa proceder de otro país, sino un constructo o etiqueta para definir colectivamente al extraño que posibilita delimitaciones sentimentales de pertenencia y cohesión de quienes lo nombran a partir de la proximidad física pero no cultural y afectiva. Es una relación paradójica de coexistencia y condicionamiento mutuo. Así, el término "extranjero", entendido como extraño o forastero (Shütz, 2012) "puede ser cualquiera que trata de ser aceptado y tolerado por un grupo al que se aproxima" (Sabido, 2012, p. 14).

De forma que los términos "extranjero", "forastero" o "extraño" connota a cualquier persona que ingresa a una entidad geográfica específica (municipio, delegación, entidad federativa o país) para residir en ella. En añadidura se refiere al inmigrante según definiciones elementales (Consejo Nacional de Población, 2012; Gabinet d'Studis Socials, Dirección General de Inmigración y Voluntariado. Consejería de Política Social, Mujer e Inmigración. Comunidad Autónoma de la Región de Murcia, 2007); cuya característica sociológica es la interacción de posibilidades múltiples acentuando lo transitorio de su posición. El rasgo más definitorio es que no coincide biográficamente su historia con la del resto del colectivo; sin embargo y a pesar de que en algún momento llegue a tener congruencias experienciales con el grupo al que arriba, 
y se conforme como parte de él, continúa teniendo un estatus de extraño; como alguien que está cerca y distante a la vez. No obstante, hay colectivos que no posibilitan ni reconocen esta característica dual del extranjero, haciendo de su contacto, relaciones negativas (Simmel, 2012). Y como se ha documentado en las obras clásicas al respecto, los entornos hostiles en contra de extranjeros o foráneos provocan el desarrollo de actitudes de desconfianza que pueden traducirse en acciones violentas (Thomas \& Znaniecki, 2006).

Existen dos tradiciones científicas sociales que dan cuenta de las razones de actitudes hostiles contra inmigrantes. Por un lado se explican a partir de teorías de la competencia por mercado de trabajo; por el otro a causa variables sociológicas, psicológicas, afectivas e ideológicas (Markaki \& Longhi, 2012).

El primer enfoque concentra los argumentos de las actitudes negativas a partir del cálculo costo-beneficio individual o grupal de beneficios materiales referidos puntualmente a los puestos de trabajo y las consecuencias distributivas del ingreso o bienes hacia potenciales nuevos miembros del colectivo (Markaki \& Longhi, 2012; Mesenguer \& Maldonado, 2015). La segunda perspectiva atiende a razones de corte sociocultural. En esta se enfatizan explicaciones de la necesidad colectiva definitoria y categorizadora de sí a partir de la construcción de la otredad como referente obligatorio y delimitante identitario, consintiendo los sentidos de pertenencia y distancia. Tal ejercicio conlleva una clasificación jerárquica del "nosotros" en la superioridad frente a los "otros" por debajo (Markaki \& Longhi, 2012); lo que ocasiona a su vez estereotipos y denominaciones lingüísticas tajantes para su identificación puntual (Fuentes \& Márquez, 2006). A partir del mantenimiento de las distancias con el uso de arquetipos como práctica generalizadora así como calificado de segunda categoría, el contacto o interacción con estos sectores demarcados como extraños, foráneos o externos desencadena actitudes (entendidas como una respuesta evaluativa en relación a objetos culturales, ideológicos o personas que tienen consecuencias cognitivas, afectivas y comportamentales [Cea , 2002]) defensivas o sensaciones de amenaza de la auto enunciación (Beck, 2007).

Las razones de las actitudes negativas $u$ hostiles se explican a partir de orientaciones de sentido individuales que responden a marcos referenciales de corte social que sustentan tal criterio (Arriaga, 2015); pero también estos referentes son políticos o legales que reflejan los marcos culturales generales que instituyen los patrones de acción y valoración (Penchaszadeh, 2008). Uno de los elementos que conjuga estas dos lógicas responde a la concepción identitaria nacional que romantiza y protege celosamente sus mitos fundacionales que explican sus orígenes ancestrales (Florence \& Martiniello, 2005) y se refleja en leyes puntuales al respecto. De forma que las explicaciones de las actitudes ante la extrañeidad vinculada a la inmigración no responde exclusivamente a un criterio económico o de competencia por los espacios laborales, sino que regularmente ambas lógicas influyen (Wimmer, 2007).

Para el caso mexicano, la conformación de su narración fundacional como país tuvo de base el recelo y desconfianza hacia lo externo, el inmigrante como estereotipo de amenaza, que se expresaron en políticas migratorias abrasivas en por lo menos tres etapas: la primera, como actitud contra la dependencia colonial, enseguida, con el pensamiento liberal en el que la distancia al colonialismo quedó de manifiesto y en tercer momento como parte de la construcción de nación a la posterior Revolución Mexicana que enarboló el mestizaje así como los orígenes indígenas (Rebolledo, 2017). Esto reflejó una importante tendencia negativa hacia la existencia de inmigrantes internacionales en el país desde la etapa independentista hasta la postrevolucionaria, ya que nunca rebasó un punto porcentual de población externa hasta los años cuarenta del siglo XX. Incluso fue hasta 2011 que la inmigración ilegal dejó de ser castigada con pena de cárcel; sin embargo, no prescinde de rasgos abiertamente violatorios de derechos humanos contra externos ya que los anula porque se subordinan a la soberanía y seguridad nacional (Gall, 2018). De forma que la tendencia hacia la abierta exclusión al agente externo, a pesar de que el porcentaje de inmigrantes internacionales no ascienda al 1\% (Gall, 2018; París, Ley, \& Peña, 2016), se mantiene casi intacta en forma de prejuicio.

Pero esta postura no solamente se limita a las o los externos, sino también a aquellas personas internas que no compatibilizan con los marcos referenciales del discurso mestizo autodefinitorio del país (Navarrete, 2016). Aunque los prejuicios se orientaron de igual manera hacia la población inmigrante interna del país en los que se documentan diversas expresiones de rechazo: por ejemplo la tendencia oficalista del México a inicios del siglo XX para orientar a la capital como epicentro de cosmopolitismo, emulando los centros europeos y rechazando a las provincias por el supuesto atraso; por ende a los y las ciudadanas provenientes de esos lugares (Monsiváis, 2006). 
Pero también esta lógica definitoria se reflejó en contracorriente; por ejemplo, el movimiento antichilango (expresión lingüística para categorizar estereotipadamente a personas que provienen de la ciudad capital de México) en la entidad de Baja California en la década de los 70 (Gruel, 2015), y el prejuicio antichilango más visible contemporáneamente en la ciudad de Guadalajara (Guerra, 2014); aunque se asegura que es un sentimiento generalizado en todo el país (Murueta \& Orozco, 2014). No obstante, la hostilidad hacia otros agentes culturales localizados geográficamente en distintos espacios, aunque en el interior del propio país, es un elemento inherente del México inicial como actual; ya que se notan estas diferencias entre miembros de colectivos diversos, por ejemplo: entre "regios y jaliciences, tijuanenses y mexicalenses, los de juaritos y los chihuahuitas, obregonenses y hermosillenses" (Cervantes, 1999).

Para el caso del Estado de Hidalgo, la inmigración es consecuencia de tres procesos. El primero por razones internas de ciudadanos(as) nacionales e internacionales que se incorporan a la entidad, que si bien no constituyen porcentajes significativos, en tiempos recientes se corrobora un crecimiento excepcional (Granados, 2010). En segundo lugar la migración de retorno desde el país vecino del norte cuya intensidad crece desde 2008 (Cruz, 2018; Granados \& Pizarro, 2013) y en tercer lugar la migración de tránsito por parte de centroamericanos(as). Este último proceso se debe a que el tren de carga que recorre de sur a norte el país (La Bestia) atraviesa la entidad (Blancas, 2015). De manera que resulta fundamental analizar su caso ya que independientemente de la recurrencia cuantitativa de inmigrantes en la entidad, las relaciones sociales tácitas resultan un importante eje de indagación. Es menester, por tanto, realizar un acercamiento sucinto del contexto. El estado de Hidalgo es uno de los treinta y dos que conforman la república mexicana. Cuenta con una superficie de $20,813 \mathrm{~km}^{2}$, que representa $1.1 \%$ del territorio nacional, distribuidos en 64 municipios, de los cuales Pachuca de Soto es el centro político administrativo. Su población en el año 2010 fue de $2,665,018$ habitantes, en los que $51.7 \%$ son varones y $48.3 \%$ mujeres; representa $2.4 \%$ de la población nacional. La distribución es $52 \%$ en localidades urbanas y $48 \%$ en localidades rurales (Franco, 2012).

La mitad de la población del estado de Hidalgo se conforma por personas jóvenes, es decir, edades entre 15 y 30 años. La esperanza de vida promedio es de 73.6 años (Fernández, 2014). El promedio de forma- ción escolar es de ocho años de estudios y el porcentaje de analfabetismo es de poco más de $6 \%$.

En este estado se ubican tres etnias indígenas: nahuas, otomíes y tepehuas; y se utilizan diez lenguas: nahua, otomí, tepehua, mixteco, zapoteco, totonaca, mazahua, maya y huasteco.

Las principales actividades económicas son el comercio, construcción, transporte y la industria alimentaria; que contribuye al PIB nacional en $1.5 \%$. Sin embargo, es importante señalar que el Estado se ubica entre las primeras entidades con mayor marginación del país y como uno de los principales estados expulsores de migrantes hacia Estados Unidos de América (Franco, 2012).

En cuanto a la inmigración, $40 \%$ de los nuevos residentes a la entidad son provenientes de la capital del país, Ciudad de México, y la entidad que la rodea, el Estado de México (Cruz, 2015). Se caracterizan por ubicarse en el rango etario de 20 y 44 años y menores de edad.

La forma en que se considera su arribo es particular; ya que, comparativamente a otras entidades federativas, este Estado se ubica entre los diez, de treinta y dos, que en mayor medida juzgan que crean desintegraciones sociales; según la Enadis (Consejo Nacional para Prevenir la Discriminación, 2011). Ya que el Estado de Morelos lo consideró en 53.3\%; en segundo lugar Chiapas con $44.10 \%$; enseguida Durango con el $41.60 \%$; puebla con $39.80 \%$; Oaxaca con $39.30 \%$; luego Veracruz con $36.80 \%$ e Hidalgo con $33.30 \%$ de encuestados(as) de la muestra. El Estado que en menor medida reportó informantes que concordaron con tal afirmación fue Nayarit con $11.60 \%$ (Figura 1). 


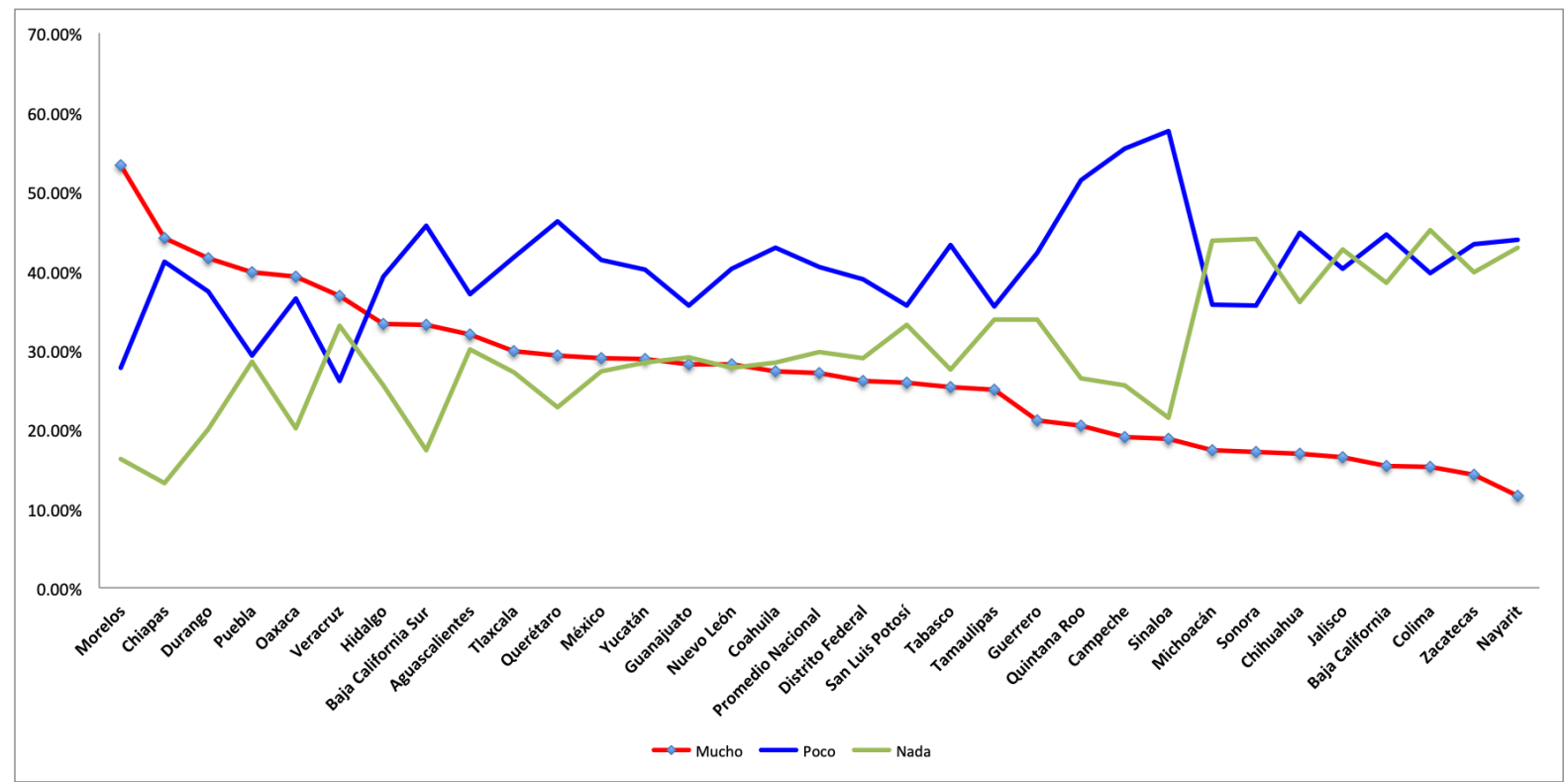

Figura 1. Porcentaje de informantes que piensa que la llegada de gente de afuera provoca divisiones entre las personas. Adaptado de la Enadis 2010 (Conapred, 2011).

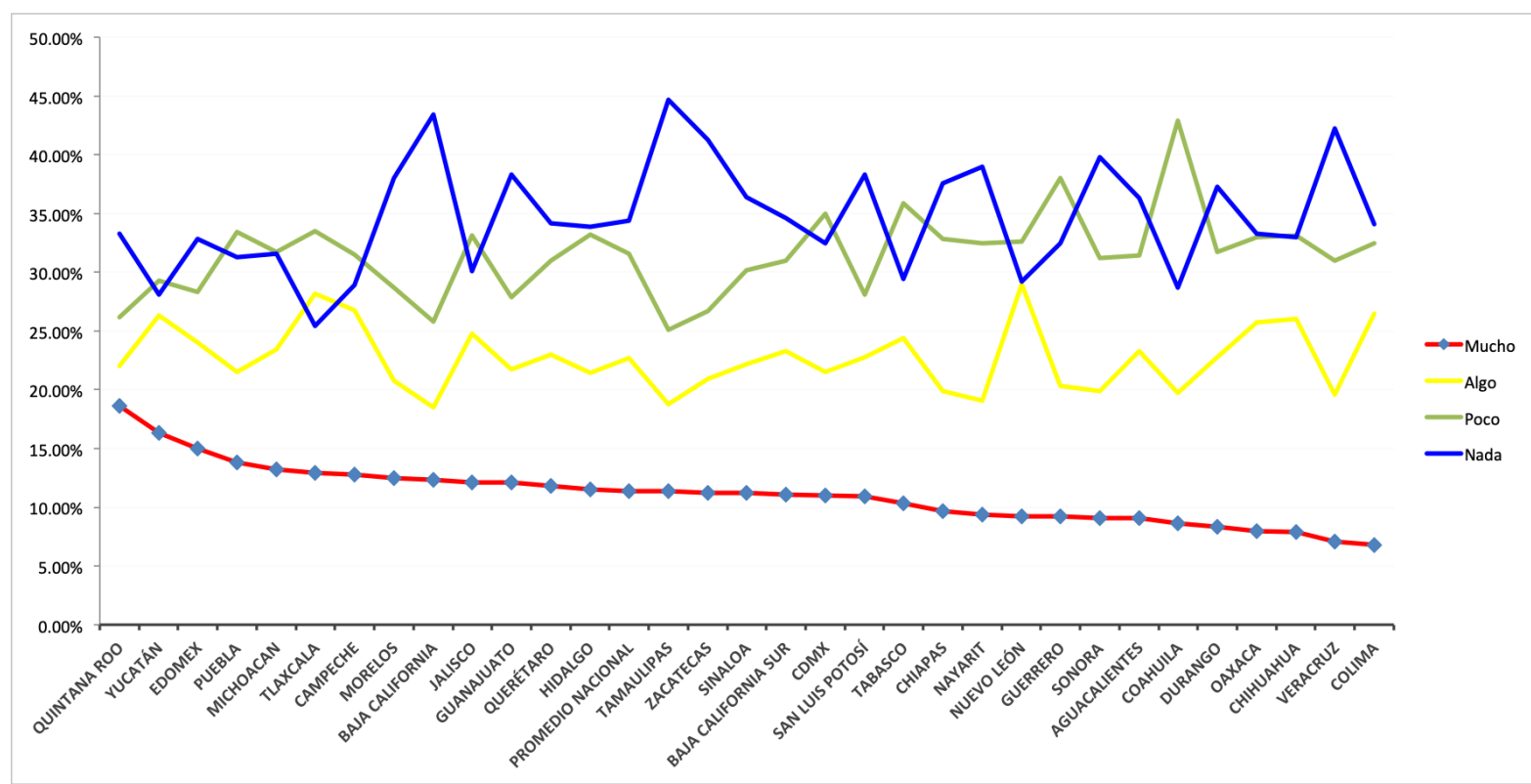

Figura 2. Porcentaje de informantes que piensa que la llegada de gente de afuera provoca divisiones entre las personas. Adaptado de la Enadis, 2017 (Instituto Nacional de Estadística y Geografía, 2017). 
El porcentaje general, a nivel nacional, reflejó un acuerdo con la postura en $27.10 \%$, colocando así a la submuestra hidalguense por arriba en 9.7 puntos porcentuales y considerado como un colectivo con altos índices de juicios discriminatorias contra los y las nuevas residentes en su demarcación.

Pero en la última encuesta (2017) se preguntó "Cuando hay conflictos entre la gente de un mismo vecindario, colonia o localidad ¿Cuánto considera que se deban a diferencias por ser originario de otro lugar?". El 11.4\% de informantes de la muestra nacional refirió que influye "Mucho", y el 22.7\%, "Algo". La entidad Quintana Roo encabeza porcentualmente en informantes con actitudes discriminatorias ya que el $18.6 \%$ de encuestados(as) refirió que se debe "Mucho" a esa condición y el 22\%, "Algo". Enseguida Yucatán con $16.3 \%$ y $26.3 \%$, Estado de México con $15 \%$ y $24 \%$, respectivamente. Con menor porcentaje se localiza Colima con $6.8 \%$ y $26.5 \%$; después Veracruz con $7.1 \%$ y $19.6 \%$. El $11.5 \%$ de personas encuestadas en Hidalgo refirió que "Mucho", el 21.4\%, "Algo". Ubicándose por lo tanto en el bloque de muestras que en mayor medida considera actitudes excluyentes, solo por encima del promedio nacional por $0.1 \%$ y $0.3 \%$ en cada categoría de la variable.

Sin embargo, en comparación entre las dos encuestas para el Estado de Hidalgo, las diferencias son mínimas; ya que en la primera el $33.3 \%$ de encuestados(as) señaló lo "Mucho" que provocan divisiones entre la gente las personas que llegan de fuera. Pero en 2017, la suma de las categorías "Mucho" y "Algo" constituyen el 32.9\%; mostrándose, así, escasos cambios en la consideración.

\section{Materiales y métodos}

Los datos para el presente análisis fueron obtenidos de la Encuesta Nacional sobre Discriminación 2010 realizada por el Conapred (2011), organismo público descentralizado que del 14 de octubre al 23 de noviembre de 2010 visitó 13,751 hogares en todo el país. La muestra fue aleatoria, poliepática, estratificada, por conglomerados y las unidades primarias de muestreo se seleccionaron con probabilidad proporcional a su población; arrojando información de 52,095 personas (Conapred, 2011). La encuesta de 2017 fue elaborada por el Instituto Nacional de Estadística y Geografía (Inegi) y Conapred del 21 de agosto al 13 de octubre de ese año, y fue aplicada a 39,101 viviendas con los mismos criterios muestrales que la anterior. De forma que se encuestaron 102,245 personas.

Para la submuestra del Estado de Hidalgo en la encuesta del 2010, se utilizaron los resultados de 688 informantes. De los cuales $51.5 \%$ son mujeres y $48.5 \%$ son varones. La media de edad es de 32 años. El porcentaje de informantes sin instrucción educativa es de $4.8 \% ; 65.4 \%$ tienen educación básica; $20.7 \%$ tiene educación media y $8.5 \%$, educación profesional. $55.1 \%$ es empleado mientras que $44.9 \%$ no labora. Para 2017, los resultados son de 2,177 casos divididos en $57.1 \%$ de mujeres y $42.9 \%$ varones, cuya media de edad es de 45.2 años. El 6.2\% carece de instrucción educativa alguna; el $26 \%$ cuenta con educación primaria; $27.2 \%$, secundaria; $16 \%$, media superior; $18 \%$, con estudios profesionales y el $1.8 \%$, con posgrado. En cuanto la situación laboral el 53.4\% tiene empleo; $25.4 \%$ se ocupa de labores domésticas; $8.9 \%$ es desempleado(a); 6.3\%, jubilado(a); 3.9\%, es estudiante y el $1.3 \%$ es impedido para laborar.

La variable dependiente a utilizar para la encuesta de 2010 versa acerca de la división entre personas en un mismo lugar, solicitando al o la informante que elija la dimensión cualitativa que más se ajuste a su opinión:

Variable O_o5.- Siempre hay diferencias entre la gente que vive en un mismo lugar ¿Qué tanto cree usted que la gente que llegue de fuera provoque divisiones entre la gente?
(1) Mucho
(2) Poco
(3) Nada

En 2017 hubo variación en la pregunta y dimensiones de la respuesta:

Variable Po2_1_6.- Cuando hay conflictos entre la gente de un mismo vecindario, colonia o localidad ¿Cuánto considera que se deben a diferencias por ser originario de otro lugar?
Mucho (2) Algo
(3) Poco
(4) Nada

Esta pregunta fue formulada al total de la submuestra y de la cual tomaremos la dimensión primera como la elemental para el análisis; ya que representa la intensidad actitudinal favorable (Cea, 2002) a la discriminatoria hacia sujetos foráneos. Entre los estudios previos que han utilizado variables semejantes tipo Likert figuran: Falomir-Pichastor, Muñoz-Rojas, Invernizzi y Mugny (2004), Laborín, Parra y Valdéz (2012) y Ponterotto y colaboradores (1995). 
Como variables independientes consideramos, en ambas encuestas, al sexo (numerada en 2010 como P7 y en 2017 con código sexo) de los y las informantes, como ejercicio de control típico. En algunos estudios se ha asegurado que es en los varones donde se reflejan mayores incidencias y tendencias de intolerancia contra los inmigrantes (Willems, 1995; Smith-Castro, 2010) pero en otros estudios se hacen advertencias que aluden a las mujeres como mayoritariamente propensas a la exclusión migratoria (Dustman \& Preston, 2007; Ponce, 2017) o como ostentadoras de posturas conservadoras al respecto (Donato, \& Perez, 2016). Sin embargo, haremos uso de la variable con la finalidad de hacer una exploración para este caso particular.

La edad, como variable (p8 en 2010 y con código edad en 2017) recodificada en diez rangos atendiendo a los parámetros que los organismos públicos mexicanos establecen como minoría de edad (hasta 17 años), jóvenes (18 a 25, 26 a 30), adultos ( 31 a 35,36 a 40, 41 a 45,46 a 50,51 a 55,56 a 60 ) y adultos mayores (60 años o más). Además, conforme a los resultados de algunos estudios empíricos, se considera la edad como un factor que puede determinar la tendencia hacia la discriminación; ya que, como señalan algunos resultados de estudios previos, la edad adulta es la más proclive a percibir o valorar a las personas foráneas como amenazas (Semyonov, Raijman, Yom, \& Schmidt, 2004). Incluso se ha llegado a afirmar puntualmente que son las personas mayores de treinta años quienes son más proclives a demostrar actitudes violentas contra los migrantes (Willems, 1995).

El grado educativo (signado como P12n en 2010 y en 2017 como niv) conforma una variable fundamental para predecir analíticamente actitudes discriminatorias, ya que de manera recurrente se utilizan en estudios semejantes (Semyonov et al., 2004) e hipotéticamente se afirma que a mayor grado educativo, los prejuicios discriminatorios son menores (Wagner $\&$ Zick, 1995). A nivel estadístico se ha afirmado una alta correlación entre sujetos propensos a la violencia contra inmigrantes y bajos niveles educativos (Mugny, Sanchez-Mazas, Roux, \& Pérez, 1991; Willems, 1995).

En cuanto al estado civil (p14 en 2010 y en 2017 p3_16), éste conforma un predictor que habilita la posibilidad de incidir en la actitud discriminatoria contra diversos grupos históricamente segregados como portadores de VIH (Genberg et.al., 2009), homosexuales y lesbianas (Herek \& Capitanio, 1995). En cuanto a inmigrantes, es usada en estudios empíricos que pre- tenden explorar con el total de variables sociodemográficas elementales (Semyonov et al., 2004; Semyonov \& Raijman, 2006) y se ha encontrado que es entre informantes casados donde se encuentran mayores prejuicios contra foráneos a causa de la preocupación del bienestar de sus familias (Ceobanu \& Escandell, 2010).

La clase social para la encuesta de 2010 fue construida a partir de recategorizar una variable cuantitativa discreta que registró ingresos brutos para convertirla en intervalos conforme los marcos referenciales que las instituciones mexicanas establecieron para definir estratos económicos. Según la Secretaría de Economía, la clase social denominada pobreza extrema se ubica en el rango de ingresos mensuales de 0.0 a 2,699 pesos mexicanos; la clase pobre, de 2,700 a 6,799 pesos; la clase media baja, de 6,800 a 11,599; la clase media, de 11,600 a 34,999 ; la clase media alta, de 35,000 a 84,999 y la clase alta con ingresos mayores a 85,000 (Aguayo, 2014; González, 2015). En la encuesta 2017 se utilizó la variable nombrada como estrato. La forma en que esta ha sido referida para explicar las tendencias de intolerancia hacia la inmigración es altamente explicativa a partir de las condiciones contextuales. Es decir, según estudios empíricos europeos, en etapas de crisis económicas, el sentimiento hacia inmigrantes es negativo u hostil (Quillian, 1995), suponiendo, entonces, que sin importar el estrato económico, la opinión antinmigrante depende de la coyuntura.

De forma específica, y prescindiendo de la variable crisis económica, se argumenta que las clases bajas son las que tienen mayores actitudes de tolerancia a la inmigración; ya que típicamente se encuentran en mayor contacto con estos nuevos residentes, mientras que las clases media-altas tipifican como riesgosas las entradas de nuevos residentes a las ciudades, a pesar de su escasa interacción directa con ellos o ellas (Solé, 2010). Sin embargo, existen estudios en los que se argumenta que los estratos sociales con mayores ingresos son los que en menor medida presentan actitudes de prejuicio ante la inmigración dado que su posición económica y su preparación para trabajos de alto nivel les otorgan un tipo de seguridad que los exime de competir laboralmente con migrantes de escasa preparación (Kunovich, 2004).

Y como última variable para la predicción se contempla el estatus de empleo (p15 en 2010 y p3_17 en 2017), en las dimensiones de empleo o desempleo. Análisis teóricos han referido que el sentimiento de 
hostilidad hacia la inmigración se deriva de la percepción de la competencia en el mercado de trabajo que genera la llegada de nueva oferta laboral (Vallas, Zimmerman, \& Davis, 2009). Por otra parte, también se ha descubierto poca relación entre la situación de empleo con la tendencia actitudinal de prejuicio negativo contra inmigrantes (Schlueter, Meuleman, \& Davidoc, 2013). Por lo tanto, testaremos la variable empíricamente.

La técnica de análisis tiene por objetivo describir la independencia entre variables categóricas y ordinales (cualitativas) para construir modelos explicativos de sus mutuas relaciones, de forma análoga al modelo de regresión o análisis de varianza como se hace con variables continuas (Batista \& Sureda, 1987). Así, esta técnica estadística se justifica por la naturaleza de las variables o categorías a analizar en su relación, simplificando los datos a través de la observación de su distribución en planos de dos dimensiones (Greenacre \& Hastie, 1987; Visauta \& Martori, 2003).

La ventaja de esta técnica radica en que prescinde de las medidas de asociación entre variables, como la chi-cuadrada o coeficientes de correlación, ya que proporciona una medida de asociación más precisa que otras técnicas multivariantes y capta la correspondencia entre las dimensiones de cada variable (Sourial et.al., 2010).

Con este procedimiento multifactorial determinamos la posición de las dimensiones de las variables a partir de propiedades valorativas en un espacio vectorial de dos dimensiones y con ello podremos señalar las relaciones a partir de las proximidades entre categorías (Lozares, López, \& Borrás, 1998; Visauta \& Martori, 2003) para interpretar y comparar perfiles analíticos (Inglehart \& Welzel, 2006).

Por último, será con el método comparativo que se analizarán las semejanzas y diferencias para extraer conclusiones (Colino, 2009), a través de la especificidades de cada caso (Mills, Van de Bunt, \& De Brujin, 2006) para con ello señalar regularidades a partir de los hallazgos y proponer modelos explicativos (Rihoux, 2006; Vigour, 2011).

\section{Resultados}

Conforme a los resultados del análisis estadístico, el modelo de correspondencias múltiples arrojó datos que permiten interpretar un perfil del sujeto discriminador de inmigrantes o foráneos en esta submuestra hidalguense en 2010. Los niveles de confianza que este cálculo supone son los que de forma elemental permiten aseverar la validez de sus resultados, ya que la prueba del valor de la inercia total o resumen del modelo refirió valores 0.969 , lo que supone que más de la mitad de las inercias son explicadas. Así, la lectura de los perfiles básicos tiene como principio y referente la dimensión de la variable dependiente en su proximidad con las dimensiones de las variables independientes. Por lo tanto, en el gráfico I se representa la conformación analítica de los perfiles.

Como apreciamos en la gráfica, la variable "sexo" resulta indistintamente considerada en el cálculo, lo que supone que ambas categorías mantienen proporcionalmente consideraciones equivalentes con respecto al fenómeno. Esto se corrobora por el coeficiente de correlación entre variables cuyo resultado es 0.053 , por lo tanto, la influencia en la conformación de las dimensiones del gráfico es de 0.031 en la primera y 0.028 en la segunda. Lo mismo sucede para la variable de situación de empleo, cuyo coeficiente de correlación es de 0.079 y su contribución a las dimensiones es de 0.154 y 0.014 respectivamente.

El resto de las variables propuestas mantiene una interacción directa con las dimensiones de la variable dependiente así como altos niveles de colaboración para la conformación de los planos de las dimensiones del gráfico. El nivel de instrucción colabora en el primero con 1.155 y 0.562 en el segundo; estado civil lo hace con 1.511 y 0.872 ; la edad con 1.187 y 0.866 y la clase social lo hace con 0.106 y 0.109 , respectivamente. Estas cifras permiten elaborar una tipología básica de informante discriminador y otro de no discriminador ya que en la variable dependiente, a pesar de contar con tres dimensiones, las referentes a "poco" o "nada" se ubican próximas en el plano y ambas con distancias significativas con la dimensión "mucho".

Con lo anterior se visibilizan dos perfiles elementales. Por un lado los informantes que poco o nada consideran que la inmigración genera divisiones entre las personas. En este perfil se ubican agentes de edades entre los 46 y 60 años, de estatus civil casados(as) y divorciados y con niveles de formación académica de posgrado.

Por otro lado, el perfil que de forma categórica se asocia con altos niveles de prejuicio negativo contra la inmigración se caracteriza por conformarse de informantes de 36 a 45 años, de estrato económico medio bajo y bajo, de estatus civil separado y de formación académica normalista; es decir de educación media superior. 
Para los datos de la encuesta de 2017 los niveles de confianza del proceso reflejan valores aceptables ya que el resumen del modelo señaló 0.585 ; es decir más de la mitad de las inercias explicadas. Al igual que en la Figura 2 referente al 2010, la variable "sexo" carece de influencia en la relación para conformar perfiles, ya que su correlación con la variable dependiente es de 0.017 y su influencia en la configuración de las dimensiones del diagrama es de 0.029 para la primera dimensión y 0.011 para la segunda. Lo mismo ocurre con la variable clase social, ya que el nivel de contribución a la conformación de dimensiones es de 0.067 y 0.073 , respectivamente. Las variables con mayor peso en el plano son la edad, que aporta 0.659 en la primera dimensión y 0.575 en la segunda; enseguida el Estado civil con 0.514 y 0.362 , situación laboral con 0.529 y 0.478 ; como último, la escolaridad con 0.507 y 0.256 .

De forma que para esta reciente encuesta el perfil de informante con prejuicios negativos hacia inmigrantes se caracteriza por pertenecer a clases sociales bajas y media baja; de edad entre 26 y 55 años, de estado civil divorciado y separación del núcleo familiar; que tiene empleo o se dedica a actividades domésticas; a pesar de que los referentes numéricos señalan poca influencia del sexo, se ubica a las mujeres del lado del espectro discriminante $\mathrm{y}$, por último, de escolaridad de educación secundaria básica y formación técnica (Figura 3).

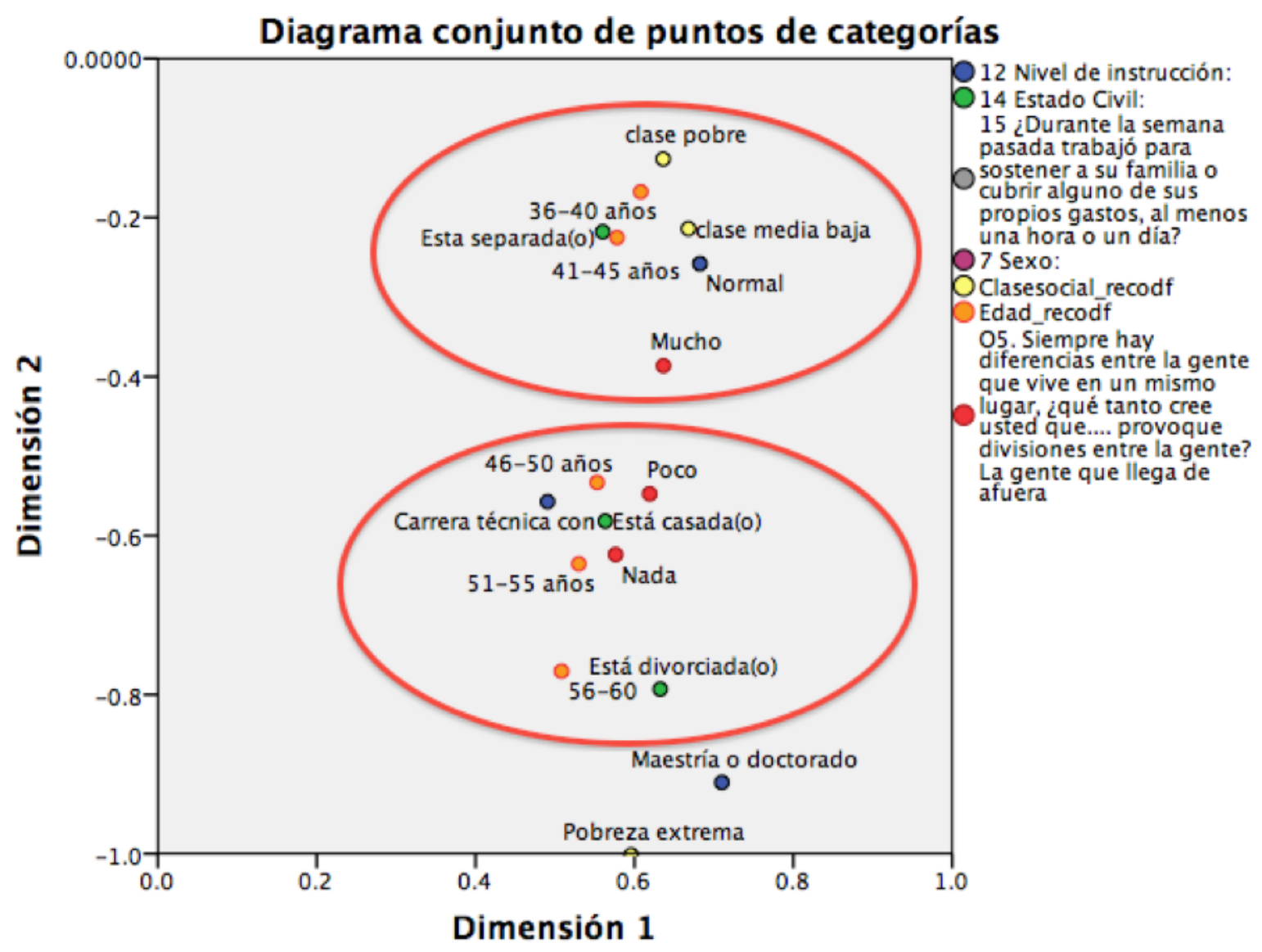

Normalización principal por variable.

Figura 3. Perfiles de informantes discriminantes y no discriminantes a partir de Análisis de Correspondencias Múltiples. Adaptado de la Enadis, 2010 (Conapred, 2011). 


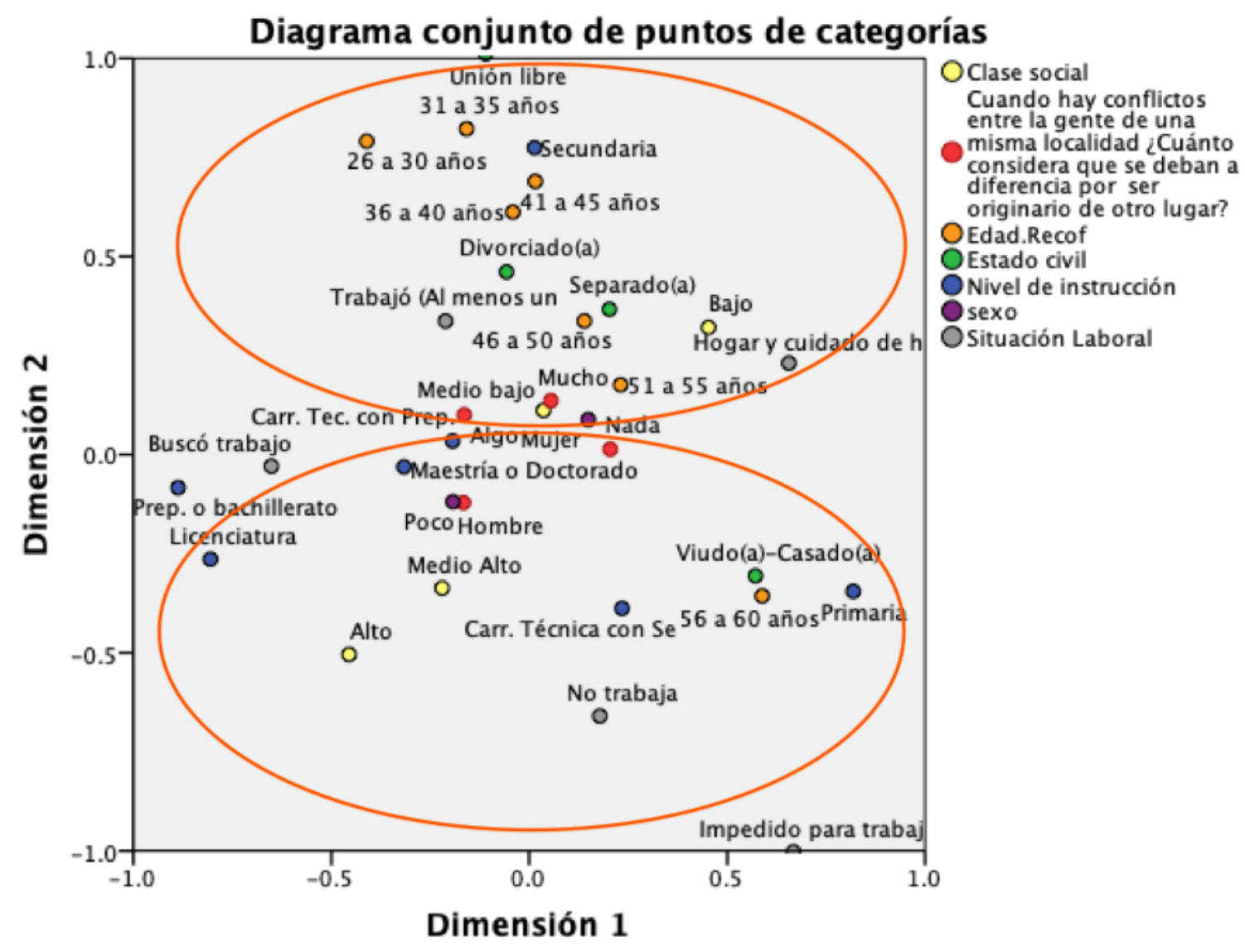

Normalización principal por variable.

Figura 4. Perfiles de informantes discriminantes y no discriminantes a partir de Análisis de Correspondencias Múltiples. Adaptado de la Enadis, 2010 (Conapred, 2011).

Mientras que el perfil de informante con actitudes no discriminatorias, por la proximidad de las dimensiones "poco" o "nada" en el diagrama, es de clase alta o media alta; de edad mayor (56 a 60 años); de estado civil casado y viudo; de nivel educativo medio superior, profesional y posgrado, y de situación laboral "No trabaja", "impedido para trabajar" y "en busca de trabajo".

\section{Discusión}

Las tradiciones teóricas y empíricas de investigación científica social típicamente consideradas para explicar el fenómeno de las actitudes antinmigrantes se han dividido en dos. La primera la explica a partir de teorías de la competencia basadas en el mercado laboral. Y la segunda prioriza explicaciones a partir de teorías y variables sociológicas, psicológicas, afectivas e ideológicas (Markaki \& Longhi, 2012). Esta investigación se adscribe a esta última vertiente ( $\sin$ dejar de lado la primera línea, considerándola solamente como un factor más de explicación); ya que su objetivo se limitó a aproximarnos a la construcción de tipologías estadísticas que permiten construir un perfil analítico de informante cuya actitud ante la inmigración es prejuiciosa negativamente.

En primera instancia reconocemos que el resultado del análisis multifactorial proyectó una dicotómica 
distancia de posturas de tolerancia e intolerancia entre los perfiles extraídos en ambos ejercicios, ubicando en posiciones contrarias la valoración ante los foráneos en la entidad; ya que la distribución de las categorías de la variable dependiente se posicionó en extremos opuestos en el diagrama. Lo anterior se interpreta, en primera instancia, como sujetos coexistentes en un contexto cuyos ordenes simbólicos tienden hacia la radicalidad. Como ejemplo histórico de ello, aludimos a un proceso surgido a mediados de 2011 en la ciudad capital del Estado (Pachuca de Soto), durante la coyuntura política preelectoral. En él se exacerbó y extendió una campaña de señalamiento negativo en contra de inmigrantes que radicaban en la ciudad y que pretendían dirigir administrativamente la metrópoli. La campaña se difundía con el eslogan "Pachuca para los pachuqueños" en el trasporte público. El Consejo Nacional para Prevenir la Discriminación consideró tal campaña como excluyente contra cualquier ciudadano(a). Por tal motivo ordenó retirarla (Notimex, 2011). De manera que la definición compartida por informantes se orienta en sentidos de valoración dicotómicas a partir de posturas excluyentes entre el "nosotros" y "los otros" como referente identitario (Beck, 2007; Sabido, 2012; Simmel, 2012).

Con respecto a la variable ocupacional resultó eximido como altamente asociado en el cálculo de 2010 o causante de actitudes hostiles, frente al resto de categorías; tal y como se ha demostrado en distintas evidencias empíricas que lo estudian como factor determinante (Hainmueller \& Hiscox, 2007). Una hipótesis basada en datos locales que pudiera explicar esa baja relación obedece a que en la capital del Estado, centro de actividades económicas y políticas de la entidad, los inmigrantes representan más de la mitad de la población. Sin embargo su participación económica, en puestos de trabajo de la ciudad en 2010, es significativamente inferior en contraste con años anteriores (Granados \& Franco, 2015). Por lo tanto, no son los puestos de trabajo en riesgo lo que influye en las actitudes antiforáneas. Y en el cálculo de 2017, las categorías de la variable ocupacional que señalaron que no trabajan se encuentran impedidos para hacerlo o se encuentran en busca de empleo, se mantienen en el perfil de informante no discriminatorio. Por ello reafirmamos la idea que los sentidos de orientación de acción y valoración contra inmigrantes no responde a razones instrumentales o de competencia ocupacional.
En el mismo tenor, la variable sexo resultó poco influyente, tal y como diversos estudios empíricos advirtieron (Krings \& Olivares, 2007; Wimmer, 2007).

Las diferencias de edades entre los perfiles, según el ejercicio multivariado de 2010, permiten asegurar que es entre agentes de edades adultas, de 36 a 45 años donde se reflejan las mayores actitudes antimigratorias, a diferencia de las generaciones más jóvenes, tal y como estudios empíricos conocidos han señalado (Card, Dustman, \& Preston, 2005; O'Rourke \& Sinnot, 2006). Sin embargo para esta submuestra, es en segmentos etarios superiores, de 46 a 55 años, que se perciben niveles de tolerancia superiores. Podría explicarse con base en que el segmento de 36 a 45 años se encuentra inmerso en plena etapa laboral, mientras que las otras no conforman o concentran del todo a la población activa. El cuidado de las seguridades materiales y laborales atraviesa la construcción valorativa (Card et al., 2005). En el cálculo de 2017, asume los mismos criterios, por lo tanto aseveramos que, tal y como se diagnostica a partir de estudios empíricos referidos, entre más edad menor actitud discriminatoria; que se corresponde con la etapa última de etapa laboral formal. Empero, habría que matizarlo mediante estudios multivariantes más profundos para resolver empíricamente esta argumentación.

En cuanto al estado civil, los resultados que encontramos refieren que, a diferencia de la literatura al respecto (Kahanec \& Tosun, 2009; Semyonov \& Raijman, 2006), el estatus marital es un factor altamente determinante de las actitudes ante la inmigración; en contraste con lo señalado en análisis previos (Brenner \& Fertig, 2006; Ceobanu \& Escandell, 2010). De forma que es es en los estados civiles de "separados", según el perfil de 2010, que se localizan los perfiles de alta exclusión contra inmigrantes. Mientras que en la última etapa se concentra en ese mismo estatus civil, pero también en divorciados y en unión libre. Por lo tanto, los informantes casados o en situaciones de formación de núcleos familiares estables o solamente disueltos por viudez son los perfiles más propensos hacia la tolerancia ante foráneos.

La clase social resultó ser una variable altamente relacionada con las actitudes antimigratorias en el perfil 2010 y 2017, siendo de manera categórica las clases más bajas las que en mayor medida lo consideran, lo cual se corresponde con los resultados de distintos estudios empíricos previos (Kunovich, 2004; Willems, 1995, 2007). La explicación de ello obedece a las posturas clásicas que refieren al mercado de trabajo como 
variable interviniente. Es decir, los informantes de clases bajas consideran la inmigración como un factor que pudiera mermar las posibilidades de obtener recursos provenientes del trabajo ya que potencializa la competencia laboral y las desventajas económicas (Polanyi, 2007). También se alude a que las clases altas poseen una perspectiva cosmopolita con respecto a la comprensión de su entorno; es decir, tienen herramientas intelectuales que les permiten ver las virtudes que miembros de otros territorios aportan y a la vez comprenden las limitaciones de su propio territorio; mientras que las clases bajas se localizan en una perspectiva parroquial, que concentran su atención en la protección absoluta de su entorno (Haubert \& Fussel, 2006).

En cuanto a la dimensión de la instrucción académica, las principales hipótesis difundidas así como hallazgos empíricos afirman una correlación entre mayores niveles de instrucción académica y actitudes favorables hacia las personas inmigrantes. Esto se debe a quienes han recibido una mayor preparación académica, al igual que la noción de cosmopolitismo, son capaces de reconocer las ventajas de la integración de foráneos en la cultura y economía local (Bobo \& Licari, 1989; Card et al., 2005; Wagner \& Zick, 1995; Willems, 1995); lo cual se corresponde con los descubrimientos de este puntual estudio en ambos perfiles. Y de manera general se ha demostrado la relación entre elevados niveles educativos con escasas actitudes de prejuicio ante cualquier agente considerado colectivamente estigmatizado (Pratto, Sidanius, Stallworth, \& Malle, 1994).

De manera que es visible la congruencia entre ambos perfiles de informante con actitudes discriminatorias en cuanto variables que los conforman: rangos de edad que típicamente se ubican en etapa laboral, de estrato económico bajo, situación civil que refiere ausencia de conformación de núcleo familiar y formación no profesional. Y para el caso del último perfil suma el rasgo laboral activo y el hogar.

\section{Conclusiones}

Con la revisión y discusión de los resultados de la exploración, las conclusiones a las que llegamos se orientan en dos sentidos. El primero consiste en señalar que desde 2010 y hasta 2017, una tercera parte de hidalguenses encuestadas(os) consideran que las personas que vienen de fuera ocasionan problemas o conflictos en la población receptora. Posicionándose, en ambos periodos, en el grupo de entidades con mayores porcentajes de informantes con actitudes excluyentes ante la inmigración en general.

En el otro sentido, formulamos perfiles analíticos de informantes tendientes hacia actitudes discriminatorias contra inmigrantes. Como revisamos en la Enadis 2010 (Conapred, 2011), se compone por sujetos de entre 36 a 45 años de edad, de clase media baja o baja, con estudios medios superiores y de estado civil separado. Mientras que en la Enadis 2017 es de clase social baja y media baja, de edades de entre 26 y 55 años, de estado civil divorciado y separación del núcleo familiar, que tienen empleo o se dedican a actividades domésticas y por último con escolaridades de educación secundaria básica y formaciones técnicas.

Con esta información se pretende hacer una contribución al estado de conocimiento empírico que pueda ser útil para la comprensión del fenómeno de exclusión hacia un sector poblacional que es creciente en la entidad. Además suponemos que la ventaja proporcionada por esta indagación exploratoria, coadyuva a incentivar ejercicios semejantes con respecto al tratamiento discriminatorio que padecen otros sectores poblacionales que la Encuesta Nacional sobre Discriminación en México detectó de forma descriptiva; y que su tratamiento técnico sea con fines de extraer distintos perfiles de agentes discriminantes para encaminar propuestas de programas de sensibilización, que aunadas a las políticas positivas y afirmativas, se orienten a una población en específico para cada caso de exclusión social.

\section{Agradecimientos}

Esta investigación exploratoria es resultado del proyecto de investigación "Diagnóstico de la discriminación en Pachuca de Soto, Hidalgo y la zona conurbada" financiado por el Programa para el Desarrollo Profesional Docente de la Secretaría de Educación Pública de México con número de registro en la Universidad Autónoma del Estado de Hidalgo UAEHEXB-148.

\section{Referencias}

Aguayo, J. (14 de mayo de 2014). Las clases sociales en México según la secretaría de economía... ¿Usted a cuál pertenece? Tribuna. Periodismo libre y objetivo. Recuperado de http://www.jorgevargas.com.mx/archivo/20140514/8635.htm 
Arriaga, R. (2015). Inmigración y discriminación: Hacia un trabajo de aplicación conceptual de la noción weberiana de sentido. Estudios Fronterizos, 16(32), 99-127. https://doi.org/10.21670/ ref.2015.32.a04

Batista, J., \& Sureda, J. (1987). Análisis de correspondencias y técnicas de clasificación: su interés para la investigación en las ciencias sociales y del comportamiento. Journal of the study of education and development. Infancia y Aprendizaje, 10(39-40), 171-186. https://doi.org/10.1080/0210 3702.1987.10822184

Beck, U. (2007). Cómo los vecinos se convierten en judíos. La construcción política del extraño en una era de modernidad reflexiva. Papers. Revista de Sociología, 84, 47-66. http://dx.doi.org/10.5565/ $\mathrm{rev} / \mathrm{papers} / \mathrm{v} 84 \mathrm{n} 0.1632$

Blancas, N. (2015). El lugar importa. Perfiles poblacionales y sociales de los lugares de tránsito del migrante centroamericano, en el tema de la migración. En M. Quezada (Coord.), Estudios demográficos en el Estado de Hidalgo (Tomo 6). Pachuca de Soto, Hidalgo: Universidad Autónoma del Estado de Hidalgo.

Bobo, L., \& Licari, F. (otoño, 1989). Education and political tolerance: Testing the effects of cognitive sophistication and target group affect. Public Opinion Quarterly. 53(3). 282-308. https://doi. org/10.1086/269154

Bonfil, G. (1987). México Profundo. Una civilización negada. México: Grijalbo.

Brenner, J., \& Fertig, M. (2006). Identifying the determinants of attitude towards immigrants: A structural cross.country analysis. IZA, (Discussion Paper, 2306).

Card, D., Dustman, C., \& Preston, I. (2005). Understanding attitudes to immigration and minority module of the first European Social Survey. Center of Research and Analysis of Migration Discussion. CReAM, (Discussion Paper, 03/05).

Cea, M. A. (2002). La medición de las actitudes ante la inmigración: Evaluación de los indicadores tradicionales de "racismo". Revista Española de Investigaciones Sociológicas, (99), 87-111.

Ceobanu, A., \& Escandell, X. (2010). Comparative analyses of public attitudes toward inmigrants and inmigration using multinational survey data:
A Review of theories and research. Annual Review of Sociology, 36, 309-328. https://doi. org/10.1146/annurev.soc.012809.102651

Cervantes, J. (22 de agosto de 1999). Ya nadie hace patria? La muerte del antichilanguismo. La Jornada. Recuperado de https://www.jornada.com. mx/1999/08/22/mas-jesusa.html

Consejo Nacional de Población. (2012). Glosario. México: Autor. Recuperado de http://www.conapo.gob.mx/work/models/CONAPO/Resource/2160/3/images/Glosario_Migracion_Interna. pdf

Consejo Nacional para Prevenir la Discriminación. (2011). Encuesta Nacional sobre Discriminación en México/ Enadis 2010. Recuperado de http:// www.conapred.org.mx/userfiles/files/Enadis2010-RG-Accss-002.pdf

Consejo Nacional para Prevenir la Discriminación, \& Secretaría de Desarrollo Social (2005). Primera Encuesta Nacional sobre Discriminación en México. Recuperado de: http://www.conapred.org. $\mathrm{mx} /$ userfiles/files/Presentacion_de_la_Encuesta_final.pdf

Constitución Política de los Estados Unidos Mexicanos, Art. primero (2016).

Cruz, I. (noviembre, 2015). Migración y desarrollo desde lo local. El Estado de Hidalgo en perspectiva. Trabajo presentado en 20 Encuentro Nacional sobre Desarrollo Regional en México, Cuernavaca, Morelos. México. Recuperado de http:// ru.iiec.unam.mx/2984/1/Eje8-030-Cruz.pdf

Cruz, I. (2018). Políticas públicas y migración de retorno desde Estados Unidos: El caso de los municipios metropolitanos hidalguenses. Tla-melaua, 12(45), 209-238. http://dx.doi.org/10.32399/ rtla.12.45.770

Donato, K., \& Perez, S. (2016). A different hue of the gender gap: Latino immigrants and political conservatism in the United States. The Russel Sage Foundation. Journal of the Social Sciences, 2(3), 98-124. https://doi.org/10.7758/RSF.2016.2.3.06 The B.E. Journal of Economic Analysis \& Policy, 7(1). https://doi.org/10.2202/1935-1682.1655

Falomir-Pichastor, J. M., Muñoz-Rojas, D., Invernizzi, F., \& Mugny, G. (2004). Perceived in-group threats as a factor moderating the influence of ingroup norms on discrimination against foreingers. 
Eureopen Jorunal of Social Psychology, 34(2), 135-153. https://doi.org/10.1002/ejsp.189

Fernández, P. (2014). Dinámica demográfica 19902010 y proyecciones de población 2010-2030. Hidalgo, México: Consejo Nacional de Población.

Florence, E., \& Martiniello, M. (2005). Social Science Reseach and Public Policies: The case of inmigrant in Belgium. International Journal of Multicultural Societies, 7(1), 49-67.

Franco, L. M. (2012). La migración en el estado de Hidalgo. Un enfoque de desarrollo regional.Hidalgo, México: Universidad Autónoma del Estado de Hidalgo.

Fuentes, C., \& Márquez, M. (Eds.). (2006). Actitudes ante la inmigración. El reflejo lingüístico. Sevilla: Junta de Andalucía, España.

Gabinet d'Studis Socials, Dirección General de Inmigración y Voluntariado. Consejería de Política Social, Mujer e Inmigración. Comunidad Autónoma de la Región de Murcia (2007). Glosario de términos de integración de inmigrantes. Murcia, España: Dirección General de Inmigración y Voluntariado. Consejería de Política Social, Mujer e Inmigración. Comunidad Autónoma de la Región de Murcia. Recuperado de http://www.carm.es/ ctra/cendoc/doc-pdf/publicaciones/2007_Glosarioinmigrantes.pdf

Gall, O. (2018). Racismos y xenofobias frente a los migrantes: 1910 - 2018. REMHU, Revista Interdisciplinaria da Mobilidade Humana, 26(53), 115-134. https://doi.org/10.1590/198085852503880005308

Genberg B., Hlavka Z., Konda,K., Maman S., Chariyalerstsak S., Chingono A., ... Celentano D. (2009). A comparison of HIV/AIDS-related stigma in four contries: Nagative attitudes and perceived acts of discrimination towards people living with HIV/AIDS. Social Science \& Medicine. 68(12). 2279-2287. https://doi.org/10.1016/j. socscimed.2009.04.005

González, J. (25 de enero de 2015). Salarios, clases sociales e informalidad. Vanguardia. Recuperado de https://www.vanguardia.com.mx/columnas-salariosclasessocialeseinformalidad-2257012.html

Granados, J. (2010). Los nuevos residentes de Pachuca: Análisis de la migración y características de los migrantes en la aglomeración urbana de Pa- chuca. Hidalgo, México: Universidad Autónoma del Estado de Hidalgo.

Granados, J., \& Franco, L. (2015). Migración y trabajo en la ciudad de Pachuca Hidalgo. En O. Díaz, J. Morales, J. Rodríguez \& J. Campos. (Coords.), El trabajo que México necesita (pp. 528-542). México: Asociación Mexicana de Estudios del Trabajo, Centro de Investigación Interdisciplinaria para el Desarrollo del Capital Humano.

Granados, J., \& Pizarro, K. (2013). Paso del norte, qué lejos te vas quedando. Implicaciones de la migración de retorno en México. Estudios Demográficos y Urbanos, 28(2), 469-496. http://dx.doi. org/10.24201/edu.v28i2.1435

Greenacre, M., \& Hastie, T. (1987). The geometric interpretation of correspondence analysis. (1987). Journal of the American Statistical Association, 82(398), 437-447.

Gruel, V. (2015). El movimiento antichilango en Baja California, México. La mirada literaria académica y periodística 1971-1991. Culturales, 3(1), 77105.

Guerra, E. (2014). La ciudad dividida. Protesta altermundista y violencia política en Guadalajara. Estudios Sociológicos, 32(96), 541-571.

Hainmueller, J., \& Hiscox, M. (2007). Educated preferences: Explaining attitudes toward immigration in Europe. International Organization, 61(2), 399-442. https://doi.org/10.1017/ S0020818307070142

Haubert, J., \& Fussell, E. (2006). Explaining pro-immigrant sentiment in the U.S.: Social class, cosmopolitanism, and percepción of immigrants. International Migration Review, 40(3). 489-507. https:// doi.org/10.1111/j.1747-7379.2006.00033.x

Herek, G., \& Capitanio, J. (1995). Black heterosexual's attitude toward lesbians and gay men in the United States. The Journal of Sex Research, 32(2). 95-105. https://doi.org/10.1080/00224499509551780

Inglehart, R., \& Welzel, C. (2006). Modernización, cambio cultural y democracia. La secuencia del desarrollo humano. Madrid, España: Centro de Investigaciones Sociológicas.

Kahanec, M., \& Tosun, M. (2009). Political economy of immigration in Germany: Attitudes and citizenship aspirations. International Migration 
Review, 43(2). 263-291. https://doi.org/10.1111/ j.1747-7379.2009.00765.x

Krings, A., \& Olivares, J. (2007). At the doorstep to employment: Discrimination against inmigrants as a function of applicant ethnicity, job type, and raters' prejudice. International Journal of Psychology, 42(6), 406-417. https://doi. org/10.1080/00207590701251721

Kunovich, R. (2004). Social structural position and prejudice: An exploration of cross-national differences in regression slopes. Social Science Research, 33(1), 20-44. https://doi.org/10.1016/ S0049-089X(03)00037-1

Laborín, J. F., Parra, E. M., \& Valdéz, G. C. (2012). Discriminación y prejuicio de jóvenes sonorenses hacia el migrante indígena. Estudios Sociales, (2), 333-347.

Ley Federal para Prevenir y Erradicar la Discriminación. (2014). Secretaría de Gobernación. Consejo Nacional para Prevenir la Discriminación. México. Recuperado de http://www.conapred.org.mx/ userfiles/files/LFPED_web_ACCSS.pdf

Lozares, C., López, A., \& Borrás, V. (1998). La complementariedad Log-lineal y del análisis de correspondencias en la elaboración de tipologías. Papers. Revista de Sociología, 55, 79-93. http:// dx.doi.org/10.5565/rev/papers. 1933

Markaki, Y., \& Longhi, S. (2012). What determines attitudes to inmigration in European countries? An Analysis at the regional level. Norface Migration. Discussion Paper, (32), 1-31. https://doi. org/10.1093/migration/mnt015

Mesenguer, C., \& Maldonado, G. (2015). Las actitudes hacia los inmigrantes en México: Explicaciones económicas y sociales. Foro Internacional, 55(3), 772-804. https://doi.org/10.24201/fi.v55i3.2279

Mills, M., Van de Bunt, G., \& De Brujin, J. (2006). Comparative research. Persistent problems and promising solutions. International Sociology, 21(5), 619-631. https://doi. org/10.1177/0268580906067833

Monsiváis, C. (2006). Pasiones urbanas a la orden. (La Ciudad de México y la cultura 1900-1950). Andes, 17, 283-411.

Mugny, G., Sanchez-Mazas, M., Roux, P., \& Pérez, J. (1991). Independence and interdependence of group judgments: Xenophobia and minori- ty influence. European Jornal of Social Psychology, 21(3), 213-223. https://doi.org/10.1002/ ejsp. 2420210304

Murueta, M., \& Orozco, M. (2014). Psicología de la violencia. Causas, prevención y afrontamiento. México: Manual Moderno.

Navarrete, F. (2016). México racista. Una denuncia. México: Grijalbo.

Notimex. (11 de agosto de 2011). Consideran discriminatoria campaña "Pachuca para los pachuqueños". SPDNoticias. Recuperado de https:// www.sdpnoticias.com/notas/2011/08/04/consideran-discriminatoria-campana-pachuca-para-los-pachuquenos

O'Rourke, K., \& Sinnot, R. (2006). The determinants of individual attitudes toward immigration. European Journal of Political Economy, 22(4), 838-861. https://doi.org/10.1016/j.ejpoleco.2005.10.005

París, M. D., Ley, M., \& Peña, J. (2016). Migrantes en México. Vulnerabilidad y riesgos. Tijuana, México: Organización Internacional para las Migraciones.

Penchaszadeh, A. P. (2008). La cuestión del extranjero. Una mirada desde la teoría de Simmel. Revista Colombiana de Sociología, (31), 51-67. http://dx. doi.org/10.15446/rcs

Polanyi, K. (2007). La gran transformación. Los orígenes políticos y económicos de nuestro tiempo. México: Fondo de Cultura Económica.

Ponce, A. (2017). Gender and anti-immigrant attitudes in Europe. Socius: Sociological Research for a Dinamic World, 3, 1-17. https://doi. org/10.1177/2378023117729970

Ponterotto, J., Burkard, A., Rieger, B., Grieger, I., D'Onofrio, A., Dubuisson, A., ... Sax, G. (1995). Development and initial validation of the quick discrimination index (QDI). Educational and Psychological Measurement, 55(6), 1016-1031. https://doi.org/10.1177/0013164495055006011

Pratto, F., Sidanius, J., Stallworth, L., \& Malle, B. (1994). Social dominance orientation: A personality variable predicting social and political attitudes. Journal of Personality and Social Psychology, 67(4), 741-763. https://doi.org/10.1037/00223514.67.4.741 
Quillian, L. (1995). Prejudice as a response to perceived group threat: Population composition and anti-immigrant and racial prejudice in Europe. American Sociological Review, 60(4), 586-611. https://doi.org/10.2307/2096296

Rebolledo, O. (2017). México: Identidad, diversidad y extranjería. Revista de Ciencias Sociales (Cr), 4(158), 45-59.

Rihoux, B. (2006). Qualitative comparative analysis (QCA) and related systematic comparative methods and remaining challenges for social sciences research. International Sociology, 21(5), 679706. https://doi.org/10.1177/0268580906067836

Rodríguez, J. (2005). Definición y concepto de la no discriminación. El Cotidiano, 134(21), 23-29.

Sabido, O. (2012). Tres miradas sociológicas ante el extrañamiento del mundo. En G. Simmel, A. Schütz, N. Elias \& M Cacciari, El extranjero. Sociología del extraño (pp. 9-17).: España: Sequitur

Schlueter, E., Meuleman, B., \& Davidoc, E. (2013). Immigrant integration policies and perceived group threat: A multinivel study of 27 western and Eastern european countries. Social Science Research, 42(3), 670-682. https://doi.org/10.1016/j. ssresearch.2012.12.001

Semyonov, M., \& Raijman, R. (2006). The raise of anti-forigner sentiment in european societies, 1988-2000. American Sociological Review, 71(3), 426-449. https://doi. org/10.1177/000312240607100304

Semyonov, M., Raijman, R., Yom, \& Schmidt, P. (2004). Population size percived threat, and exclusión: a multiple-indicators analysis of attitudes toward foreingers in Germany. Social Science Research, 33(4). 681-701. https://doi.org/10.1016/j. ssresearch.2003.11.003

Simmel, G. (2012). El extranjero. Sociología del extraño. España: Sequitur.

Simmel, G. (2014). Sociología. Estudios sobre las formas de socialización. México: Fondo de Cultura Económica.

Smith-Castro, V. (2010). Experiencias de discriminación social de inmigrantes Nicaragüenses en Costa Rica: Reacciones afectivas y atribuciones causales. Interamerican Journal of Psychology, 44(2), 368-381.
Sourial, N., Wolfson, C., Zhu, B., Quail, J., Fletcher, J., Karunananthan, S., ... Bergman, H. (2010). Correspondence analysis is a useful tool to uncover the relationships among categorical variables. Journal of Clinical Epidemiology, 63(6). 638-646. https://doi.org/10.1016/j.jclinepi.2010.04.003

Thomas, W., \& Znaniecki, F. (2006). El campesino polaco en Europa y América. Madrid, España: Centro de Investigaciones Sociológicas y Boletín Oficial del Estado.

Vallas, S., Zimmerman, E., \& Davis S. (2009). Enemies of the state? Testing three models of anti-immigrant sentiment. Research in Social Stratification, 27(4), 201-217. https://doi.org/10.1016/j. rssm.2009.10.002Reading in methodology. African perspectives (pp. 215-248). Senegal: Council for the Development of Social Research in Africa.

Visauta, B., \& Martori, J. (2003). Análisis estadístico con SPSS para Windows. Vol. II. Estadística multivariante. Madrid: Mc Graw Hill.

Wagner, U., \& Zick, A. (1995). The relation of formal education to ethnic prejudice: Its reliability, validity and explanation. European Journal of Social Psychology 25(1). 41-56. https://doi.org/10.1002/ ejsp. 2420250105

Willems, H. (1995). Right-wing extremism, racism or youth violence? Explaining violence against foreingers in Germany. Journal of Ethnic and Migration Studies. 21(4), 501-523. https://doi.org/10.1080/1369183X.1995.9976509

Willems, H. (2007). Development, patterns and causes of violence against foreingers in Germany: Social and bographical characteristics of perpetrators and the process of escalation. Terrorism and Political Violence, 7(1), 162-181. https://doi. org/10.1080/09546559508427288 20(1), 17-41. https://doi.org/10.1080/01419870.1997.9993946 